\title{
ECONOMIC DIFFERENCE SCHEMES FOR A HYPERBOLIC SYSTEM OF EQUATIONS WITH COMPOUND DERIVATIVES AND THEIR APPLICATION TO EQUATIONS IN THE THEORY OF ELASTICITY*
}

\author{
A. A. SAMARSKII \\ Moscow \\ (Received 1 October 1964)
}

1. IN this section we consider first of all additive difference schemes (see [1] $-[5]$ ) for a system of second order hyperbolic equations which contain compound derivatives. There are schemes with variable directions which are absolutely stable and convergent at least with a speed $O\left(|h|^{2}+\tau\right)$, where $|h|^{2}=\sum_{\alpha=1}^{p} h_{\alpha}^{2}, h_{\alpha}$ is the step in the variable $a_{\alpha}$ and $p$ is the number of dimensions. The numerical algorithm consists of the conversion of a three-point triangular operator, which reduces to the successive application of known formulae. The number of operations to determine a solution for a new time layer is proportional to the number of nodes of the space network and is a quantity of the same order as the number of operations for a purely explicit scheme. Thus the schemes put forward below are economic.

To construct economic schemes for an equation of the form

$$
\frac{\partial^{2} u}{\partial t^{2}}+\sum_{\alpha, \beta=1}^{p} A_{\alpha \beta}(t) u=f
$$

we use the common property of the operator

$$
A=\sum_{\alpha, \beta=1}^{p} A_{\alpha \beta}
$$

- Zh. vȳchisl. Mat, mat. Fiz. 5, 1, 34-43, 1965. 
i.e. its representation in the form of the sum of operators $A_{\alpha \beta}$ of simpler structure.

Additive economic schemes for a general second order hyperbolic system are then used to describe economic schemes for a system of equations in the theory of elasticity in the case of two or three space variables $(p=2, p=3)$.

A resolving scheme is also constructed for equations of elasticity, which is absolutely stable and converges with a speed $O\left(|h|^{2}+\tau^{2}\right)$. With regard to economy the resolving scheme is comparable with additive schemes, but for convergence it requires more smoothness for the solution of the differential equation.

In Section 7 an iteration scheme with alternating directions for the solution of a difference problem, corresponding to a stationary problem in the theory of elasticity, is considered.

The convergence of this scheme is proved for $p=2,3$, and it is shown that the number of iterations $v=O\left(h^{-2(p-1) ; p} \ln (1 / \varepsilon)\right)$, where $\varepsilon$ is the required accuracy.

Economic schemes of another type are considered in the two-dimensional case $(p=2$ ) for a dynamic problem of the theory of elasticity in [6] and for a static problem in the theory of elasticity in [7].

2. Let $\bar{G}=G+\Gamma=\left\{0 \leqslant x_{\alpha} \leqslant l_{\alpha}, \alpha=1, \ldots, p\right\}$ be a $p$-dimensional parallelepiped with boundary $\Gamma$. In the cylinder $\bar{Q}_{T}=\bar{G} \times[0 \leqslant t \leqslant T]$ there is a solution of the problem

$$
\begin{gathered}
\frac{\partial^{2} \mathbf{u}}{\partial t^{2}}=\sum_{\alpha, \beta=1}^{p} L_{\alpha \beta} \mathbf{u}+\mathbf{f}(x, t), \quad L_{\alpha \beta} \mathbf{u}=\frac{\partial}{\partial x_{\alpha}}\left(k_{\alpha \beta}(x, t) \frac{\partial \mathbf{u}}{\partial x_{\beta}}\right) \\
\left.\mathbf{u}\right|_{\Gamma}=v(x, t), \quad 0 \leqslant t \leqslant T, \quad \mathbf{u}(x, 0)=v_{0}(x) \\
\frac{\partial \mathbf{u}}{\partial t}(x, 0)=v_{1}(x), \quad x \in \bar{G} .
\end{gathered}
$$

Here $x=\left(x_{1}, \ldots, x_{p}\right) ; \mathbf{u}=\mathbf{u}(x, t)=\left(u^{1}, \ldots, u^{s}, \ldots, u^{n}\right), \mathbf{f}, \mathbf{v}, v_{0}, v_{1}$ are vectors of dimensionaly $n$, and $k=\left(k_{\alpha \beta}\right)=\left(k_{\alpha \beta}{ }^{s m}\right), s, m=1, \ldots, n$, is a partitioned $p \times p$ matrix with $n \times n$ submatrices, which satisfies the symmetry condition

$$
k_{\alpha \beta}^{* n}(x, t)=k_{\beta \alpha}^{m *}(x, t)
$$


and the condition of positive definiteness

$$
\sum_{s, m=1}^{n} \sum_{\alpha, \beta=1}^{p} k_{\alpha \beta}^{s m}(x, t) \xi_{\beta} m \xi_{\alpha^{n}} \geqslant c_{1} \sum_{s=1}^{n} \sum_{\alpha=1}^{p}\left(\xi_{\alpha^{s}}\right)^{2}, \quad(x, t) \in \bar{Q}_{T},
$$

where $\xi_{\alpha}=\left(\xi_{\alpha^{1}}, \ldots, \xi_{\alpha}^{s}, \ldots, \xi_{\alpha}^{n}\right) \neq 0$ is an arbitrary real vector and $c_{1}$ is a positive constant.

We shall assume that the problem (1) - (2) has a unique solution $u=u(x, t)$, which is continuous in $\bar{Q}_{T}$ and differentiable as many times as necessary for this work. To infer the a priori evaluations it is assumed that $k_{\alpha \beta}(x, t)$ satisfies a Lipschitz condition with respect to $t$ and $x_{\alpha^{\prime}}, \alpha^{\prime}=1, \ldots, p$.

The system of equations in the theory of elasticity

$$
\frac{\partial^{2} \mathbf{u}}{\partial t^{2}}=\mu \Delta \mathbf{u}+(\lambda+\mu) \operatorname{grad} \operatorname{div} \mathbf{u}+\mathbf{f}(x, t)
$$

where $\Delta \mathbf{u}=\sum_{\alpha=1}^{p} \partial^{2} \mathbf{u} / \partial x_{\alpha}{ }^{2} \quad$ is the Laplace operator, $\mathbf{u}=\left(u^{1}, \ldots, u^{p}\right)$, $\lambda=$ const $>0$ and $\mu=$ const $>$ are Lamé' s coefficients, is obviously a particular case of the system of equations (1) with $n=p$ and

$$
k_{\alpha \beta}^{s m}=\mu \delta_{\alpha \beta} \delta_{s m}+(\lambda+\mu) \delta_{\alpha s} \delta_{\beta m}, \quad \delta_{i j}= \begin{cases}1, & i=j, \\ 0, & i \neq j,\end{cases}
$$

where $\delta_{i j}$ is the Kronecker delta. Condition (3) is satisfied automatically. We shall show that condition (4) is also satisfied if $c_{1}=\mu$. In fact

$$
\begin{aligned}
\sum_{s, m=1}^{p} \sum_{\alpha, \beta=1}^{p} k_{\alpha \beta}^{m} \xi_{\alpha^{s} \xi_{\beta} m}=\mu \sum_{\alpha, s=1}^{p}\left(\xi_{\alpha^{s}}\right)^{2}+(\lambda+\mu) \sum_{\alpha, s=1}^{p} \xi_{\alpha^{\alpha} \xi_{s}}= \\
=\mu \sum_{\alpha, s=1}^{p}\left(\xi_{\alpha^{s}}\right)^{2}+(\lambda+\mu)\left(\sum_{\alpha=1}^{p} \xi_{\alpha^{\alpha}}\right)^{2} \geqslant \mu \sum_{\alpha, s=1}^{p}\left(\xi_{\alpha}\right)^{2}
\end{aligned}
$$

3. Let us introduce the difference networks $\omega_{\tau}=\left\{t_{j}=j \tau \in[0, T]\right.$, $j=0,1, \ldots\} \quad$ and $\quad \bar{\omega}_{h}=\left\{x_{i}=\left(i_{1} h_{1}, \ldots, i_{\alpha} h_{\alpha}, \ldots, i_{p} h_{p}\right) \Subset \bar{G}=G+\Gamma\right.$; $\left.i_{\alpha}=0,1 \ldots, N_{\alpha}, h_{\alpha}=l_{\alpha} / N_{\alpha}, \alpha=1,2, \ldots, p\right\}$ with steps $\mathrm{T}$ for the 
variable $t$ and $h_{\alpha}$ for the variable $x_{\alpha,} \alpha=1, \ldots, p$ : let $\gamma_{h}=\left\{x_{i} \in \Gamma\right\}$ be the boundary of the network $\bar{\omega}_{h}, \bar{\omega}_{h} \backslash \gamma_{h}=\left\{x_{i} \in G\right\}$ be the set of inner nodes, $|h|^{2}=\sum_{\alpha=1}^{p} h_{\alpha}^{2}$. Following [1], we snall introduce the notation

$$
\begin{gathered}
\mathbf{y}=\mathbf{y}\left(x_{i}, t_{j+1}\right)=\mathbf{y}^{j+1}, \quad \check{y}=\mathbf{y}^{j}, \\
x_{i}^{\left( \pm 1_{\alpha}\right)}=\left(i_{1} h_{1}, \ldots, i_{\alpha-1} h_{\alpha-1},\left(i_{\alpha} \pm 1\right) h_{\alpha}, i_{\alpha+1} h_{\alpha+1}, \ldots, i_{p} h_{p}\right), \\
\mathbf{y}^{\left( \pm 1_{\alpha}\right)}=\mathbf{y}\left(x_{i}^{\left( \pm 1_{\alpha}\right)}, t_{j+1}\right), \quad \mathbf{y}_{\bar{x}_{\alpha}}=\left(\mathbf{y}-\mathbf{y}^{\left(-1_{\alpha}\right)}\right) / h_{\alpha}, \quad \mathbf{y}_{x_{\alpha}}=\left(\mathbf{y}^{\left(+1_{\alpha}\right)}-\mathbf{y}\right) / h_{\alpha} .
\end{gathered}
$$

We shall replace the operator

$$
L_{\alpha \beta} \mathbf{u}=\frac{\partial}{\partial x_{\alpha}}\left(k_{\alpha \beta}(x, t) \frac{\partial \mathbf{u}}{\partial x_{\beta}}\right)
$$

in the difference network wh by the same scheme of the second order of approximation as in [2], assuming that

$$
\begin{aligned}
& \Lambda_{\alpha \beta} \mathbf{y}=\frac{1}{2}\left[\left(a_{\alpha \beta} \mathbf{y}_{\bar{x}_{\beta}}\right) x_{\alpha}+\left(a_{\alpha \beta}^{(+1 \beta)} \mathbf{y}_{x_{\beta}}\right)_{x_{\alpha}}\right] \text { for } \beta \neq \alpha, \\
& \Lambda_{\alpha \alpha} \mathbf{y}=\left(a_{\alpha \alpha} \mathbf{y}_{\bar{x}_{\alpha}}\right) x_{x_{\alpha}}
\end{aligned}
$$

where $\left(a_{\alpha \beta}\right)$ is a matrix-functional of the matrix $\left(k_{\alpha \beta}\right)$ with pattern $\left\{-1 \leqslant s_{\beta} \leqslant 0, \beta=1, \ldots, p\right\}$. The coefficients $a_{\alpha \beta}=\left(a_{\alpha \beta}^{8 m}\right)$ satisfy the conditions

$$
a_{\alpha \beta}^{s m}=a_{\beta \alpha}^{m s}
$$

for sufficiently small $|h| \leqslant h_{0}$, the condition

$$
\sum_{s, m=1}^{n} \sum_{\alpha, \beta=1}^{p} a_{\alpha \beta}^{s m_{\beta}} \xi^{m} \xi_{\alpha}{ }^{s} \geqslant c_{1}, \sum_{\alpha=1}^{p} \sum_{s=1}^{n}\left(\xi_{\alpha}\right)^{2}, \quad(x, t) \in \bar{\omega}_{h} \times \omega_{\tau},
$$

where $c_{1}{ }^{\prime} \leqslant c_{1}$ is a positive constant which does not depend on the network, and the condition obtained from (10) after replacing $a_{\alpha \beta}^{s m}$ by the coefficinet $\left(a_{\beta}^{s m}\right)^{\left(1_{\beta}\right)}$ at the point $\left.x_{i}^{(+1} \beta\right)$.

In the case of constant coefficients, $k_{\alpha \beta}=$ const., obviously $a_{\alpha \beta}=k_{\alpha \beta} \quad$ and instead of (8) we obtain

$$
\Lambda_{\alpha, \mathrm{y}}=\frac{1}{2} k_{\alpha \beta}\left(\mathbf{y}_{\bar{x}_{\beta} x_{\alpha}}+\mathbf{y}_{\bar{x}_{\alpha} x_{\beta}}\right), \quad \Lambda_{\alpha \alpha} \mathbf{y}=k_{\alpha \alpha} \mathbf{y}_{x_{\alpha} x_{\alpha}} ;
$$


condition (10) is satisfied on any network.

Note. For $\Lambda_{\alpha \beta} y$ instead of (8) we can consider other representations also, e.g.

$$
\begin{aligned}
& \Lambda_{\alpha \beta} y=\frac{1}{2}\left[\left(a_{\alpha \beta} \mathbf{y}_{\bar{x}_{\beta}}\right)_{\bar{x}_{\alpha}}+\left(a_{\alpha \beta}^{\left(+1_{\beta}\right)} \mathbf{y}_{x_{\beta}}\right) x_{\alpha}\right] \\
& \Lambda_{\alpha \beta} y=\frac{1}{2}\left[\left(a_{\alpha \beta} \mathbf{y}_{\bar{x}_{\beta}}\right)_{\dot{x}_{\alpha}}+\left(a_{\alpha \beta}^{\left(+1_{\beta}\right)} y_{x_{\beta}}\right){ }_{x_{\alpha}}\right],
\end{aligned}
$$

where $v_{x_{\alpha}}=\frac{1}{2}\left(v_{x_{\alpha}}+v_{x_{\alpha}}\right)$ is the central difference derivative. In all cases the condition (10) will be satisfied for a sufficiently small step $|h| \leqslant h_{0}$ and all the subsequent conclusions retain their validity.

4. Let us introduce the "triangular" operators $L^{-}$and $L^{+}$. For this we write the symmetrical matrix $k_{\alpha \alpha}=\left(k_{\alpha \alpha}^{\infty m}\right)$ in the form of the sum of two triangular matrices $k_{\alpha \alpha}=k_{\alpha \alpha}^{-}+k_{\alpha \alpha}^{+}, k_{\alpha \alpha}^{-}=\left(k_{\alpha \alpha}^{-s m}\right), k_{\alpha \alpha}^{+}=\left(k_{\alpha \alpha}^{+s m}\right)$, assuming $k_{\alpha \alpha}^{-s s}=k_{\alpha \alpha}^{+s s}=\frac{1}{2} k_{\alpha \alpha}^{s s}, k_{\alpha \alpha}^{-s m}=k_{\alpha \alpha}^{s m}, k_{\alpha \alpha}^{+s m}=0$ if $m<s, k_{\alpha \alpha}^{+s m}=$ $k_{\alpha \alpha}^{s m}, k \alpha \alpha=0$ with $m>s$ and any $\alpha=1, \ldots, p$. The matrix $k_{\alpha \alpha}^{\mp}$ is a diagonal $p \times p$ matrix with submatrices which are triangular $n \times n$ matrices, conjugate to each other

$$
k_{\alpha \alpha}^{-s m}=k_{\alpha \alpha}^{+m s} \quad\left(a_{\alpha \alpha}^{-s m}=a_{\alpha \alpha}^{+m s}\right)
$$

In accordance with the representation $k_{\alpha \alpha}=k_{\alpha \alpha}^{-}+k_{\alpha \alpha}^{+}$we obtain

$$
L_{\alpha \alpha}=L_{\alpha \alpha}^{-}+L_{\alpha \alpha}^{+}, \quad \Lambda_{\alpha \alpha}=\Lambda_{\alpha \alpha}^{-}+\Lambda_{\alpha \alpha}^{+},
$$

where

$$
\stackrel{\mp}{L_{\alpha \alpha} \mathbf{u}}=\frac{\partial}{\partial x_{\alpha}}\left(\stackrel{\mp}{k_{\alpha \alpha}} \frac{\partial \mathbf{u}}{\partial x_{\alpha}}\right)
$$

etc. In view of condition (13) the operators $\Lambda_{\alpha \alpha^{-}}$and $\Lambda_{\alpha \alpha^{+}}$are conjugate to each other on the network $\omega_{h}$ in the sense of the scalar product

$$
(\mathbf{y}, \mathbf{v})=\sum_{\omega_{h}} \mathbf{y}\left(x_{i}\right) \mathbf{v}\left(x_{i}\right) H, \quad H=h_{1} \ldots h_{p},
$$

i.e.

$$
\left(\Lambda_{\alpha \alpha}^{-} \mathbf{y}, \mathbf{v}\right)=\left(\Lambda_{\alpha \alpha}^{+} \mathbf{v}, \mathbf{y}\right), \quad a=1, \ldots p
$$


where $y$ and $v$ are arbitrary network functions, vanishing on the boundary $\gamma_{h}$ of the network $\omega_{h}$ *

We shall put the operator

$$
L=\sum_{\alpha, \beta=1}^{p} L_{\alpha \beta}
$$

in the form of the sum of two triangular operators

$$
\begin{gathered}
L=L^{-}+L^{+}, \quad L_{\mp}=\sum_{\alpha, \beta=1}^{p} L_{\alpha \beta}^{\mp}=\sum_{\alpha=1}^{p} L_{\alpha}^{\mp}, \quad L_{\alpha} \mp=\sum_{\beta=1}^{p} L_{\alpha \beta}^{\mp}, \\
L_{\alpha \beta}^{\mp}=L_{\alpha \alpha}^{\mp} \text { if } \beta=\alpha, \quad L_{\alpha \beta}=L_{\alpha \beta \beta}, \\
L_{\alpha \beta}^{+}=0 \text { if } \beta \leqslant \alpha, \quad L_{\alpha \beta}^{+}=L_{\alpha \beta}, \\
L_{\alpha \beta}=0 \text { if } \beta>\alpha, \quad L_{\alpha}^{-}=L_{\alpha \alpha}+\sum_{\beta=1} L_{\alpha \beta}, \quad L_{\alpha}=L_{\alpha \alpha}+\sum_{\beta=\alpha+1}^{p} L_{\alpha \beta} .
\end{gathered}
$$

By virtue of the principle of additivity of $[1-5]$ the solution of the system of equations

$$
\frac{\partial^{2} \mathbf{u}}{\partial t^{2}}=\sum_{\alpha=1}^{p}\left[\left(L_{\alpha}^{-}+L_{\alpha}^{+}\right) \mathbf{u}+\mathbf{f}_{\alpha}\right], \quad \sum^{p} \mathbf{f}_{\alpha}=\mathbf{f}
$$

reduces to the successive solution, on an $\omega_{h} \times \omega_{T}$ network with step $T / p$, of the simpler equations

$$
\frac{1}{p} \frac{\partial^{2} \mathbf{u}}{\partial t^{2}}=L_{\alpha}^{-} \mathbf{u}+L_{\alpha}^{+} \mathbf{u}+\mathbf{f}_{\alpha}
$$

The case where $L_{\alpha \beta}=\delta_{\alpha \beta} L_{\alpha \alpha,}$, i.e. compound derivatives are absent, is considered in [1].

We now introduce the values $\mathbf{y}^{j+a / p}=\mathbf{y}_{(\alpha)}$, intermediate between $\mathbf{y}^{j}=\check{y}$ and $y^{j+1}=\mathbf{y}$ assuming that $y^{(j-1)+a / p}=\check{y}_{(\alpha)}$, and use for the determination of $y_{(\alpha)}$ a difference scheme which approximates equation (16) with number $\alpha$. By analogy with [1], for the approximation of $\partial^{2} u / \partial t^{2}$ we use the $(p+1)$-th time layer

$$
\frac{1}{p} \frac{\partial^{2} \mathbf{u}}{\partial t^{2}} \sim \sigma_{p} \mathbf{u}_{\tau_{\alpha} \bar{t}_{\alpha}}, \quad \alpha=1, \ldots, p
$$




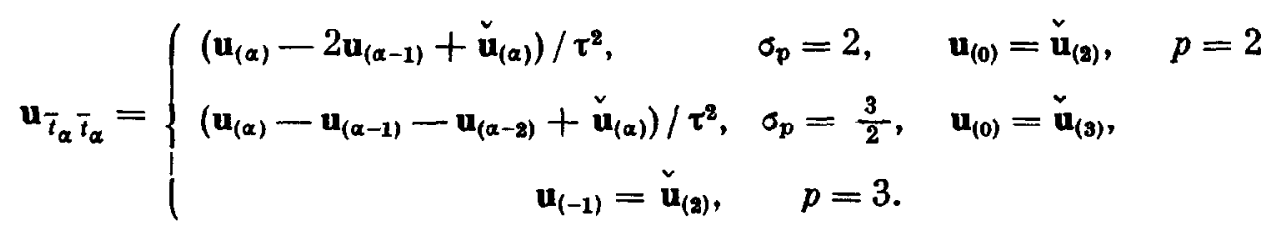

The additive scheme of alternating directions for the problem (1) - (2) will have the form

$$
\sigma_{p} \mathbf{y}_{\bar{t}_{\alpha} \bar{t}_{\alpha}}=\sum_{\beta=1}^{\alpha} \Lambda_{\alpha \beta}^{-} \mathbf{y}_{(\beta)}+\sum_{\beta=\alpha}^{p} \Lambda_{\alpha \beta}^{+} \tilde{y}_{(\beta)}+\varphi_{\alpha}, \quad \alpha=1, \ldots, p, \quad(x, t) \in \omega_{h} \times \omega_{t},
$$

$$
\mathbf{y}_{\alpha}=v\left(x, t_{j}^{*}\right) \text { with } x_{\alpha}=0, l_{\alpha}, \quad \alpha=1, \ldots, p ; \quad \mathrm{y}(x, 0)=v_{0}(x)
$$

where $\varphi_{\alpha}=\varphi_{\alpha}\left(x, t_{j}{ }^{*}\right)$ is a second order approximation on the network $\omega_{h}$ of the function $f_{\alpha}(x, t), t_{j}^{*} \in\left[t_{j}, t_{j+1}\right]$, e.g. $t_{j}^{*}=t_{j+1 / 2}=t_{j}+0.5 \tau$. The coefficients $\alpha_{\alpha \beta}=\alpha_{\alpha \beta}\left(x, t_{(\alpha) \cdot)}^{*}\right)$ are taken at the middle moment $t_{(\alpha)}=t_{j}+\frac{\alpha}{p} \tau-0.5 \tau \quad$ (cf. [1]).

The second initial condition can be approximated by analogy with [1], or more simply by assuming

$$
\mathbf{y}^{\alpha / p}=v_{1}(x)+\frac{\alpha \tau}{\rho} \widetilde{v}_{1}(x), \quad \alpha=1, \ldots, p-1, \quad p=2,3 .
$$

Such a condition is sufficient for an accuracy $O\left(\tau+|h|^{2}\right)$. Let us rewrite (18) in the form

$$
\left(E-\frac{\tau^{2}}{\sigma_{p}} \Lambda_{\alpha \alpha}^{-}\right) \mathbf{y}_{(\alpha)}=H_{\alpha}(\mathbf{y})+\frac{\tau^{2}}{\sigma_{p}} \sum_{\beta=1}^{\alpha-1} \Lambda_{\alpha \beta} \mathbf{y}_{(\beta)}+\mathbf{F}_{\alpha}=\boldsymbol{\Phi}_{\alpha}
$$

where

$$
\mathbf{F}_{\alpha}=\frac{\tau^{2}}{\sigma_{p}}\left[\sum_{\beta=\alpha}^{p} \Lambda_{\alpha \beta}^{+} \check{y}_{(\beta)}+\varphi_{\alpha}\right]
$$

$R_{\alpha}(\mathbf{y})=2 \mathbf{y}_{(\alpha-1)}-\check{\mathbf{y}}_{(\alpha)}$ if $p=2, R_{\alpha}(\mathbf{y})=\mathbf{y}_{(\alpha-1)}+\mathbf{y}_{(\alpha-2)}-\check{\mathbf{y}}_{(\alpha)}$ if $p=3$ and $E$ is the operator of identity.

Here it is obvious that to determined $y_{(\alpha)}$ (all $y_{(\beta)}$ for $\beta<\alpha$ and all $\check{y}_{(\beta)}$ for $\beta=1, \ldots, p$ are already known) we obtain a system of three- 
point equations with a triangular matrix for their coefficients. Its solution reduces to an inversion of the operator $E-\left(\tau^{2} / \sigma_{p}\right) \Lambda_{\bar{a}}$, which is attained by an $n$-fold use of the ordinary formulae for each chain $y(\alpha)$ (see [1]) for fixed $\alpha=1, \ldots, p$. To realize the algorithm (21) we must bear in mind the values of $y_{(\alpha)}$ on $p$ layers.

Let us write the equation for the $s$-th components $y_{(\alpha)}{ }^{8}$ of the vector $\mathbf{y}(\alpha)$

$$
y_{(\alpha)}^{z}-\frac{\tau^{2}}{\sigma_{p}}\left(a_{\alpha \alpha}^{-s 8} y_{x_{\alpha}}^{s}\right)_{x_{\alpha}}=\Phi_{(\alpha)}^{8}+\frac{\tau^{2}}{\sigma_{p}} \sum_{m=1}^{s-1}\left(a_{\alpha \alpha}^{-s m} y_{x_{\alpha}}^{m}\right)_{x_{\alpha}}
$$

Here $\Phi_{\alpha}$ is known, since the calculation takes place in the direction from $\alpha$ to $\alpha+1, \alpha=1, \ldots, p$; the second term is also known if we determine successively $y_{(\alpha)}, \ldots, y_{(\alpha)}, \ldots, y_{(\alpha)}{ }^{p}$, i.e. carry out the calculation from $s$ to $s+1$. Hence it is obvious that we can find $y_{(\alpha)}{ }^{s}$ by solving the first boundary value problems for the three-point equations on segments parallel to the axis $O x_{\alpha}$.

If we interchange the roles of $L_{\alpha^{-}}$and $L_{\alpha^{+}}$, respectively,

$$
\Lambda_{\alpha}{ }^{-}=\Lambda_{\alpha \alpha}^{-}+\sum_{\beta=1}^{\alpha-1} \Lambda_{\alpha \beta}=\sum_{\beta=1}^{\alpha} \Lambda_{\alpha \beta}^{-} \text {and } \Lambda_{\alpha}^{+}=\Lambda_{\alpha \alpha}^{+}+\sum_{\beta=\alpha+1}^{p} \Lambda_{\alpha \beta}=\sum_{\beta=\alpha}^{p} \Lambda_{\alpha \beta}^{+}
$$

we obtain a second additive scheme

$$
\sigma_{p} \mathbf{y}_{\bar{t}_{\alpha} \bar{t}_{\alpha}}=\sum_{\beta=\alpha}^{p} \Lambda_{\alpha \beta}^{+} \mathbf{y}_{(\beta)}+\sum_{\beta=1}^{\alpha} \Lambda_{\alpha \beta}^{+} \check{\mathbf{y}}_{(\beta)}+\varphi_{\alpha}
$$

with the same initial and boundary conditions as in the first scheme. Here to determine $y_{\alpha}$ we must invert the triangular three-point operator $E-\left(\tau^{2} / \sigma_{p}\right) \Lambda_{\alpha \alpha^{+}}$. Here the calculation proceeds from $\alpha+1$ to $\alpha$ and from $s+1$ to $s$.

The alternation of these two schemes gives a third scheme. Introducing the intermediate value $y^{j+\alpha / 2 p}, \alpha=1, \ldots, 2 p-1$, we obtain (cf. $[2,5])$

$$
\sigma_{p} \mathbf{y}_{\tau_{\alpha} \bar{t}_{\alpha}}=\sum_{\beta=1}^{\alpha} \Lambda_{\alpha \beta}^{-} \mathbf{y}_{(\beta)}+\sum_{\beta=\alpha}^{p} \Lambda_{\alpha \beta}^{+} \dot{\mathbf{y}}_{(\beta)}+\varphi_{\alpha}, \quad \alpha=1, \ldots, p
$$




$$
\begin{aligned}
\sigma_{p} \mathrm{y}_{\bar{t}_{\alpha^{\prime}} \bar{t}_{\alpha^{\prime}}}=\sum_{\beta=\alpha}^{p} \Lambda_{\alpha \beta}^{+} \mathbf{y}_{\left(\beta^{\prime}\right)}+\sum_{\beta=1}^{\alpha} \Lambda_{\alpha \beta^{\prime}} \stackrel{\nu}{\mathbf{y}}_{\left(\beta^{\prime}\right)}+\varphi_{\alpha^{\prime}}, \\
\alpha^{\prime}=2 p+1-\alpha, \quad \beta^{\prime}=2 p+1-\beta ;
\end{aligned}
$$

where $\quad \alpha^{\prime}=p+1, \ldots, 2 p, \quad \varphi_{\alpha^{\prime}}=\varphi_{\alpha}, \quad \alpha=p, p-1, \ldots, 2,1$.

5. Schemes (18) and (23) are stable for sufficiently small $|h| \leqslant h_{i_{0}}$, ensuring that the requirement (10) of the positive definiteness of the matrices $\left(a_{\alpha \beta}\right)$ and $\left(a_{\alpha \beta}^{(+1 \beta)}\right)$ for any $T$ is satisfied, and converge at least with a speed $O\left(|h|^{2}+\tau\right)$. The proof of these statements is carried out by analogy with $[1,4]$ by the method of energy inequalities.

Here the basic part is played by an identity of the form

$$
\sum_{\alpha=1}^{p}\left\{\left(a_{\alpha \beta}^{-} \xi_{\beta}, \xi_{\alpha}-\check{\xi}_{\alpha}\right)+\left(a_{\alpha \beta}^{+} \check{\xi}_{\beta}, \xi_{\alpha}-\check{\xi}_{\alpha}\right)=J-\check{J}(1+O(\tau))+R,\right.
$$

where

$$
\begin{aligned}
& J=\sum_{\alpha=1}^{p} \sum_{\beta=1}^{\alpha}\left(a_{\alpha \beta}^{-} \xi_{\beta}, \xi_{\alpha}\right)=\sum_{\alpha=1}^{p} \sum_{\beta=\alpha}^{p}\left(a_{\alpha \beta}^{+} \xi_{\beta}, \xi_{\alpha}\right), \\
& R=\sum_{\alpha=1}^{p}\left\{\sum_{\beta=1}^{\alpha}\left(a_{\alpha \beta}^{+} \check{\xi}_{\beta}, \xi_{\alpha}\right)-\left(a_{\alpha \beta}^{-} \xi_{\beta}, \xi_{\alpha}\right)\right\} .
\end{aligned}
$$

Using (3) it is not difficult to see that $R=0$.

Note. In the case of constant coefficients. $k_{\alpha \beta}=$ const., the given values of the accuracy of the schemes considered, (18) and (23), are valid for any $h_{\alpha}$ and $T$.

6. We now turn to the equations of elasticity (5). In this case as we have seen in Section $2, n=p$, and $k_{\alpha \beta}^{m}=$ const.,

$$
k_{\alpha \beta}^{s m}=\mu \delta_{\alpha \beta} \delta_{s m}+(\lambda+\mu) \delta_{a s} \delta_{\beta m}, \quad \delta_{s m}= \begin{cases}1, & s=m, \\ 0, & s \neq m .\end{cases}
$$

For the equations of elasticity (5) we can use any of the schemes considered in section 4 , bearing in mind that $a_{\alpha \beta}=k_{\alpha \beta}$, where $k_{\alpha \beta}$ is determined by formula (25).

We shall write in more detail the difference equations (18) in the 
case where $p=2 ;$ here $\mathbf{y}=\left(y^{(1)}, y^{(2)}\right)$ and

$$
\begin{aligned}
& 2 y_{\bar{t}_{1} \bar{t}_{1}}^{(1)}=\frac{1}{2}(\lambda+2 \mu)\left(y_{(1)}^{(1)}{\overline{x_{1}}}_{x_{1}}+\check{y}_{(1)}^{(1) \bar{x}_{1} x_{1}}\right)+\frac{1}{2}(\lambda+\mu)\left(\check{y}_{(2)}^{(2)} \bar{x}_{2} x_{1}+\check{y}_{(2) x_{2} \bar{x}_{1}}^{(2)}\right)+\varphi_{(1)}^{(1)} \\
& 2 y_{\bar{t}_{1} \bar{t}_{1}}^{(2)}=\frac{1}{2} \mu\left(y_{(1) \bar{x}_{1} x_{1}}^{(2)}+\check{y}_{(1) \bar{x}_{1} x_{1}}^{(2)}\right)+\varphi_{(1)}^{(2)}, \\
& 2 y_{\bar{t}_{2} \bar{t}_{2}}^{(1)}=\frac{1}{2} \mu\left(y_{(2) \bar{x}_{2} x_{2}}^{(1)}+\check{y}_{(2) \bar{x}_{2} x_{2}}^{(1)}\right)+\varphi_{(2)}^{(1)}, \\
& 2 y_{\bar{t}_{3} \bar{t}_{3}}^{(2)}=\frac{1}{2}(\lambda+2 \mu)\left(y_{(2) \bar{x}_{2} \bar{x}_{2}}^{(2)}+\check{y}_{(2) \bar{x}_{2} x_{2}}^{(2)}\right)+\frac{1}{2}(\lambda+\mu)\left(y_{(1) \bar{x}_{2} x_{1}}^{(1)}+y_{(1) \bar{x}_{1} x_{2}}^{(1)}\right)+\varphi_{(2)}^{(2)} .
\end{aligned}
$$

We must remember that here the upper index means the number of the component and the lower one the number of the vector $\left(y_{(1)}^{(1)}=\left(y^{(1)}\right)^{j+1 / 2}\right.$, $y_{(2)}^{(1)}=\left(y^{(1)}\right)^{j+1}=y^{(1)}, \quad \check{y}_{(2)}^{(2)}=\left(y^{(2)}\right)^{j+1}=\check{y}^{(2)} \quad$ etc.). Using (18) and (25) it is not difficult to write down the scheme for $p=3$.

7. For the equations of elasticity we can also construct a series of resolving schemes which are absolutely stable, economic and convergent with speed $O\left(\tau+|h|^{2}\right)$ or $O\left(\tau^{2}+|h|^{2}\right)$.

Let us first consider the two multidimensional schemes

$$
\begin{aligned}
& \mathbf{y}_{\bar{t} \bar{t}}=\Lambda^{-} \mathbf{y}+\Lambda^{+\check{y}}+\check{\varphi} \\
& y_{\bar{t} \bar{t}}=\Lambda^{+} \mathbf{y}+\Lambda^{-\check{y}}+\check{\varphi},
\end{aligned}
$$

where $\Lambda^{-}$and $\Lambda^{+}$are triangular operators, which approximate the triangular differential operator $L^{-}$and $L^{+}, y=y^{j+1}, \stackrel{\hbar}{y}=y^{j-1}, \grave{y}=y^{j}$.

Let $\grave{\Lambda}_{\alpha} y=y_{\vec{x}_{\alpha} x_{a}}$, and $\grave{\Lambda}_{s k}$ denote the difference approximation of the compound derivative $\partial^{2} \mathbf{u} / \partial x_{s} \partial x_{k}$, e.g. $\AA_{s k} \mathbf{y}=\frac{1}{2}\left(\mathbf{y}_{\bar{x}_{s} x_{k}}+\mathbf{y}_{x_{s} \bar{x}_{k}}\right)$ or $\AA_{8 k} y=\frac{1}{2}\left(y_{\bar{x}_{s} \bar{x}_{k}}+y_{x_{s} x_{k}}\right)$. Then the expressions for the triangular operators $\lambda^{-}$and $\Lambda^{+}$can be written in the form (the upper index $s$ or $k$ is the number of the component)

$$
\begin{aligned}
& \left(\Lambda^{-} y\right)^{s}=\frac{1}{2} \sum_{\alpha=1}^{p} x_{s \alpha} \Lambda_{\alpha} y^{s}+(\lambda+\mu) \sum_{k=1}^{m} \Lambda_{s k} y^{k}, \quad x_{s \alpha}=\mu+(\lambda+\mu)+\delta_{s \alpha} \\
& \left(\Lambda^{+} y\right)^{s}=\frac{1}{2} \sum_{\alpha=1}^{p} x_{s \alpha} \AA_{\alpha} y^{s}+(\lambda+\mu) \sum_{k=0+1}^{p} \AA_{s k} y^{k}
\end{aligned}
$$

The boundary conditions on $\gamma_{h}$ are exactly satisfied 


$$
\left.\mathbf{y}\right|_{v_{h}}=\boldsymbol{v}(x, t) \text {, }
$$

and the initial conditions have the form

$$
\mathbf{y}(x, 0)=\mathbf{y}_{0}(x), \quad \mathbf{y}_{\bar{t}}(x, \tau)=\mathbf{y}_{1}(x)+\tau \tilde{y}_{1}(x),
$$

where $\tilde{v}_{1}(x)$ is chosen so that the initial condition $\partial u / \partial t=v_{1}$ is approximated with accuracy $O\left(T^{2}\right)$; for this, for instance, it is sufficient to assume that $\tilde{v}_{1}=-\left.(L \mathbf{u}+\mathbf{f})\right|_{t=0}$.

Each of the schemes $\mathrm{I}^{-}$and $\mathrm{I}^{+}$is absolutely stable and has an accuracy $O\left(\tau+\left|h^{2}\right|\right)$. Applying these schemes alternately (e.g. scheme $(2 f)$ on odd and scheme (27) on even layers), we obtain an accuracy $O\left(\tau^{2}+|h|^{2}\right)$.

Following the principle given in (8), we shall write the generating scheme $\mathrm{II}^{-}$for the scheme $\mathrm{I}^{-}$

$$
\begin{aligned}
& A^{s} y_{\hat{t}}^{s}=\check{\Phi}^{s}+F^{s}, \quad A^{s}=\prod_{\alpha=1}^{p} A_{\alpha}^{s}, \quad A_{\alpha}^{s}=E-0.5 \tau^{2} \varkappa_{\beta \alpha} \dot{\Lambda}_{\alpha}, \\
& \check{\Phi}^{s}=\check{y}_{\bar{t}}^{s}+\tau\left[\left(\Lambda^{+} \check{y}\right)^{s}+0.5 \sum_{\alpha=1}^{p} x_{s \alpha} \AA_{\alpha} \check{y}^{8}+\check{\varphi}^{s}\right], \\
& F^{s}=\tau(\lambda+\mu) \sum_{k=1}^{s-1} \Lambda_{s k} y^{k}
\end{aligned}
$$

Similarly the generating scheme $\mathrm{II}^{+}$is written for the scheme $\mathrm{I}^{+}$. In this case only the formulae for $\check{\Phi}^{s}$ and $F^{s}$ are changed

$$
\check{\Phi}^{s}-\check{y}_{\bar{t}}^{s}+\tau\left[\left(\Lambda^{-\check{y}}\right)^{s}+0.5 \sum_{\alpha=1}^{p} x_{s \alpha} \check{\Lambda}_{\alpha} \check{y}^{s}+\check{\varphi}^{s}\right], \quad F^{s}=\tau(\lambda+\mu) \sum_{k=s+1}^{p} \check{\Lambda}_{s k} y^{k} .
$$

To determine $y^{s}$ on a new layer we can write some numerical algorithms for alternating directions. We give for scheme $\mathrm{II}^{-}$only the algorithm which we put forward in [8] for the equation of heat conduction: this algorithm has the form

$$
A_{1}^{s} v_{(1)}^{s}=\check{\Phi}^{s}+F^{s}, \quad \begin{gathered}
A_{\alpha} v_{(\alpha)}^{s}=v_{(\alpha-1)}^{s}, \quad \alpha=1,2, \ldots, p, s=1, \ldots, p ; \\
y^{s}=\check{y}^{s}+\tau v_{(p)}^{s} .
\end{gathered}
$$

We shall take the boundary conditions with $x_{\alpha}=0, x_{\alpha}=l_{\alpha}$ for $v_{(\alpha)}{ }^{8}$ in the form 
$v_{(\alpha)}^{s}=A_{\alpha+1}^{s} \ldots A_{p}^{s} v_{\tilde{t}}^{s}$ for $x_{\alpha}=0, \quad x_{\alpha}=l_{\alpha}, \quad \alpha=1, \ldots, p-1, \quad v_{(p)}^{8}=v_{\bar{t}}^{s}$

The order of calculation is as follows: the components $y^{(1)}, \ldots, y^{(p)}=$ $y^{j+1}$ are determined in turn.

For the algorithm which corresponds to scheme II $^{+}$the formulae (30) remain unchanged, but the order of calculation is reversed: the components $y^{(p)}, \ldots, y^{(1)}$ are determined successively.

Alternating the schemes $\mathrm{II}^{-}$and $\mathrm{II}^{+}$we find the solution of the problem with accuracy to within $O\left(|h|^{2}+\tau^{2}\right)$. This evaluation is obtained by the method of $[1,2,8]$.

From the formulae for $v_{(\alpha)}{ }^{s}$ it is obvious that the solution $y^{s}$ of the difference problem is determined by means of successive inversion of the triangular matrices (according to the formulae given in [9]). Therefore the resolving schemes are economic: to calculate the vector $y^{j+1}$ operations of the order $O\left(p^{2} / h^{p}\right)$ are required.

8. Te turn now to a stationary problem of the theory of elasticity

$$
L \mathbf{u}=\mu \Delta \mathbf{u}+(\lambda+\mu) \operatorname{grad} \operatorname{div} \mathbf{u}=-\mathbf{f}(x), \quad x \in G,\left.\quad \mathbf{u}\right|_{\mathbf{r}}=\mathbf{v}(x)
$$

Its solution reduces to the solution of the difference problem for establishing the parabolic system of equations

$$
\frac{\partial \mathbf{u}}{\partial t}=L \mathbf{u}+f(x), \quad \mathbf{u} \mid \mathbf{r}=v(x)
$$

with arbitrary initial data

$$
\mathbf{u}(x, 0)=v_{0}(x)
$$

For this we make use of the resolving scheme. Let the difference scheme for (32) take the form

$$
\Lambda \mathbf{v}+\Lambda^{+} \mathbf{v}=\varphi,\left.\quad \mathbf{v}\right|_{v_{h}}=v_{0}(x)
$$

We write the generating scheme for the determination of $y=y\left(x_{i}\right.$, $(j+1) \tau$ ), where $j+1$ is the number of the iteration and $\tau$ the iteration parameter, as

$$
A^{s} y_{\bar{t}}^{s}=\check{\Phi}^{s}+F^{s}, \quad A^{s}=\prod_{\alpha=1}^{p} A_{\alpha}^{s}, \quad A_{\alpha}^{s}=E-0.5 \tau x_{s \alpha} \AA_{\alpha},
$$




$$
\begin{gathered}
\check{\Phi}^{s}=\sum_{\alpha=1}^{p} x_{B \alpha} \AA_{\alpha} \check{y}^{s}+(\lambda+\mu) \sum_{k=8+1}^{p} \AA_{s k} \check{y}^{k}+\varphi^{s} \\
F^{s}=(\lambda+\mu) \sum_{k=1}^{s-1} \AA_{s k} y^{k}
\end{gathered}
$$

In this case the numerical algorithm for alternating directions of Section 6 takes the form

$$
\begin{gathered}
A_{1}^{s} w_{(1)^{s}}=\Phi^{2}+F^{s}, \quad A_{\alpha^{s}} w_{(\alpha)}^{s}=w_{(\alpha-1),}^{s} \quad \alpha>1, \\
y^{s}=\check{y}^{s}+\tau w_{(p)^{s}},\left.\quad w_{(\alpha)}\right|_{\gamma_{h}}=0,
\end{gathered}
$$

i.e. for $w_{(\alpha)}{ }^{8}$ the boundary conditions are always zero.

For comparison we quote one more numerical algorithm (two-layered)

$$
\begin{gathered}
A_{1}^{s} y_{(1)}^{s}=\tau\left(\Phi^{s}+F^{s}\right)+A^{s} y^{s}, \quad A_{\alpha}^{s} y_{(\alpha-1)}^{s}=y_{(\alpha-1)}^{s}, \quad a>1, \\
y_{(\alpha)}^{s}=A_{(\alpha+1)}^{s} \ldots A_{x}^{s} v^{s} \quad \text { for } \quad x_{a}=0, \quad x_{\alpha}=l_{\alpha}, \quad \alpha=1,2, \ldots, p-1: \\
y_{\left.(p)^{s}\right|_{\gamma_{h}}=v^{s} .}
\end{gathered}
$$

The order of the computation is the same as before: initially the first component $y^{(1)}$ is determined, then the second $y^{2}(s=2)$ etc.

To find $y^{s}$, at all nodes of the network $\omega_{h}$ operations of the order $O\left(1 / h_{1} h_{2} \ldots h_{p}\right)$ are required. The iteration process converges for $\tau=O\left(h_{*}\right), h_{*}=$ min $h_{\alpha}$. The rate of convergence is determined by the number of iterations $v \approx O\left(\left(1 / h_{*}\right) \times \ln (1 / \varepsilon)\right)$ for $p=2$ and $O\left(\left(1 / h_{*}{ }^{4 / 3}\right) \ln (1 / 8)\right)$ for $p=3$, where $\varepsilon$ is the required accuracy.

The evaluation for the number of iterations is obtained by the method of energy inequalities by analogy with $[10,7,4]$.

Switching the roles of the operators $\Lambda^{-}$and $\Lambda^{+}$, we obtain the second iteration scheme $\mathrm{II}^{+}$. The same value is obtained for the rate of convergence of the iterations with it as for the scheme $\mathrm{II}^{-}$described above. It is hoped that this is the basis which the interchange of these two iteration algorithms $\mathrm{II}^{-}$and $\mathrm{II}^{+}$can bring to speeding up to convergence. 


\section{REFERENCES}

1. SAMARSKII, A.A., Local one-dimensional difference schemes for multidimensional hyperbollc equations in an arbitrary region. Zh. vȳchisl. Mat. mat. Fiz. 4, 4,638 - 649, 1964.

2. SAMARSKII, A.A., Economic difference schemes for parabolic equations with compound derivatives. Zh, vÿchisl. Mat, mat. Fiz. 4, 4, 753$759,1964$.

3. SAMARgxiI, A.A., Economic difference method for the solution of a multi-dimensional parabolic equation in an arbitrary region. $Z h$. vychisl. Mat, mat, Fiz., 2, 5, 787-811, 1962.

4. SAMARSKII, A.A., Local one-dimensional schemes on non-uniform networks. Zh. vīchisl. Mat. mat, Fiz, 3, 3, 431-466, 1963.

5. SAMARSKII. A.A., Economic difference schemes for systems of parabolic equations. Zh. vīchist. Mat. mat. Fiz., 4, 5, $927-930,1964$.

6. KONOVALOV. A.N., Application of resolving methods to the numerical solution of dynamic problems in the theory of elasticity. $Z h$. vychisl. Mat. mat. Fiz, 4, 4, 760-764, 1964.

7. KONOVALOV, A.N., Iteration scheme for the solution of static problems in the theory of elasticity. Zh. vychisl. Mat. mat. Fiz., 4, 5, $942-945,1964$.

8. SAMARSKII, A.A. Schemes for increasing the order of accuracy for a multi-dimensional equation of heat conduction. Zh. vychisl. Mat. wat. Fix., 3, 5, 812-840, 1963.

9. BEREZIN, I.S. and ZHIDKOV, N.P., Numerical Methods (Metody vychisleni1), Vol. 2, Fizmatgiz, Moscow, 1960.

10. LEES, M. . A note on the convergence of alternating direction methods. Matb. Comput., 16, 77, 70- 75, 1962. 